\title{
PIBIC-EM NO NÚCLEO DE HISTÓRIA E ARQUEOLOGIA DA UNICAMP
}

\author{
Maria Aparecida de Andrade Almeida ${ }^{1}$ \\ Tobias Vilhena de Moraes $^{2}$
}

\section{RESUMO}

O Programa Institucional de Bolsas de Iniciação Científica para o Ensino Médio (PIBIC-EM), financiado pelo CNPq, teve início em 2008. Foi criado para despertar jovens talentos provenientes de escolas públicas brasileiras e tem oportunizado a formação de inúmeros jovens estudantes em todo o país. Especificamente no Laboratório de Arqueologia Pública (LAP) da Unicamp, jovens alunos têm participado de algumas atividades desenvolvidas pelos profissionais pesquisadores ali locados. O presente artigo visa apresentar sucintamente este projeto criado pelo CNPq e algumas das atividades acadêmicas que foram desenvolvidas no Laboratório de Arqueologia Pública da Unicamp.

Palavras-chave: Arqueologia; Iniciação Científica; Arqueologia Pública; Jovens; PIBIC-EM

\section{ABSTRACT}

The Institutional Program for Scientific Initiation Scholarships for High School (PIBIC-EM), funded by CNPq, began in 2008. It was created to awaken young talents from Brazilian public schools and has offered the formation of numerous young students throughout all the country. Specifically in the Laboratory of Public Archaeology (LAP) at Unicamp, young students have participated in some activities developed by professional researchers. This article aims to briefly present this project created by CNPq and some academic activities that were developed in the Laboratory of Public Archaeology at Unicamp.

Keywords: Archeology; Scientific research; Public Archaeology; Young people; PIBIC-EM

\section{RESUMEN}

El Programa Institucional de becas para Iniciación Científica para la escuela secundaria (PIBIC-EM), financiado por el CNPq, comenzó en 2008. Fue creado para despertar a los jóvenes talentos de las escuelas públicas brasileñas y tiene posibilitado la formación de numerosos jóvenes estudiantes en todo el país. Específicamente, en el Laboratorio de Arqueología Pública (LAP) de la Unicamp, los jóvenes estudiantes han participado en algunas de las actividades desarrolladas por los investigadores profesionales. Este artículo

\footnotetext{
1 Pós-Doutoranda em História e Arqueologia pela Universidade Estadual de Campinas (2014/Atual); Doutora e Mestre em Ciências da Religião/ Literatura e Religião no Mundo Bíblico pela Universidade Metodista de São Paulo (2006/20012); Bacharel em Teologia pela Faculdade Dehoniana de Taubaté (2002/2005); Tem experiência nas áreas Teológica e Bíblica, atuando nos campos Educacional e Pastoral; Integrante do Grupo de Pesquisa Arqueologia da Repressão e da Resistência; Bolsista FAPESP; email: mcidalmeida@hotmail.com
}

2 Pós-doutorando LAP/UNICAMP/FAPESP e Arqueólogo IPHAN-RS. Contato:
tovilhena@yahoo.com.br




\section{Revista de Arqueologia Pública}

tiene como objetivo presentar brevemente este proyecto creado por el CNPq y algunas actividades académicas que se han desarrollado en el Laboratorio de Arqueología Pública de la Unicamp.

Palabras-clave: Arqueología; Iniciación Científica; Arqueología Pública; jóvenes; PIBIC-EM

\section{O Programa PIBIB-EM}

O PIBIC-EM ${ }^{3}$ (Programa Institucional de Bolsas de Iniciação Científica para o Ensino Médio) é um programa operacionalizado pelas instituições de ensino e pesquisa (Universidades, Institutos de Pesquisa e Institutos Tecnológicos [CEFETs e IFs]) que possuem PIBIC (Programa Institucional de Bolsas de Iniciação Científica) e/ou PIBITI (Programa Institucional de Bolsas de Iniciação em Desenvolvimento Tecnológico e Inovação) para desenvolverem um Programa de educação científica que integre os estudantes das escolas de nível médio, públicas do ensino regular, escolas militares, escolas técnicas, ou escolas privadas de aplicação. As instituições de ensino e pesquisa são as responsáveis pelas cotas de bolsas de Iniciação Científica Júnior para o Ensino Médio, concedidas pelo CNPq, e cabem a elas pleitear uma cota de bolsas ao CNPq.

\section{O Programa PIBIC-EM na UNICAMP}

Na UNICAMP, o projeto PIBIC-EM ${ }^{4}$ (antigo Programa de Iniciação Científica Júnior - PIC Jr) foi criado em 2008, financiado pelo CNPq (Conselho Nacional de Desenvolvimento Científico e Tecnológico), cujo objetivo principal é dar a oportunidade para que alunos do Ensino Médio da rede pública de Campinas possam participar de atividades acadêmicas. Os estudantes são selecionados no final do primeiro semestre, e, desde que não estejam no último semestre do Ensino Médio, podem ter suas bolsas renovadas anualmente. Todos os anos são selecionados 300 estudantes. São escolhidos os alunos que obtiveram melhores resultados na escola, e estes são enviados para diversos locais da UNICAMP, a fim de que colaborem com o desenvolvimento dos trabalhos que ali são realizados. 0

\footnotetext{
${ }^{3}$ Todas as informações sobre a criação e desenvolvimento do programa PIBIC-EM foram tiradas dos sites http://www.cnpq.br/web/guest/pibic-ensino-medio e www.prp.unicamp.br/pibic-em

${ }^{4}$ http://www.prp.unicamp.br/index.php/2013-11-14-12-15-48/2013-11-14-17-32-51
} 


\section{Revista de Arqueologia Pública}

objetivo é despertar jovens talentos provenientes de Escolas Públicas que estejam regularmente cursando o $1^{\circ}$ e $2^{\circ}$ ano do ensino médio e ser indicado pela sua escola para as áreas de pesquisa científica, envolvendo-os com os desafios atuais da ciência e com a metodologia do trabalho científico.

Todos os selecionados recebem um auxílio mensal de ajuda de custo de $R \$ 100$, 00 (cem reais), assim como vale-transporte, vale-alimentação, seguro e assistência médica e odontológica para emergências, utilização de ônibus (fretado), caso haja disponibilidade; acesso às bibliotecas (SALAMÃO, 2010: 1). Para isso, os selecionados precisam realizar uma carga horária que não deve ultrapassar oito horas semanais. Devem também entregar um relatório ao final de cada semestre, assim como apresentar um pôster no Congresso Interno de Iniciação Científica da UNICAMP $^{5}$, no qual contam suas experiências e as atividades que realizaram. Cabe ao local que os recebeu incentivá-los a participarem de eventos acadêmicos.

Além disso, a Pró-Reitoria de Pesquisa concede aos orientadores um apoio financeiro no valor de $R \$ 4.000,00$ (quatro mil reais) na rubrica de material de consumo nacional ou prestação de serviços, quando o projeto acolhe, no mínimo, 3 bolsistas. A concessão ocorre pelo FAEPEX (além da cota normal anual do docente), ou aditivo FAPESP nos casos em que o docente responsável tenha projeto Regular ou Temático vigente na FAPESP, ou ainda coordenador de Centro de Pesquisa, Inovação e Difusão CEPID.

A duração do programa é de 12 (doze) meses, começando em agosto indo até julho do ano seguinte. Os alunos desenvolvem projetos coordenados por docentes ou pesquisadores da UNICAMP e submetidos para avaliação por uma comissão especialmente designada pela Pró-Reitoria de Pesquisa. O desenvolvimento do projeto de pesquisa deverá ser realizado com uma periodicidade mínima de 8 (oito) horas semanais, em dias e horários a serem definidos pelo orientador.

Visa oferecer aos docentes, estudantes de graduação e pós-graduação da UNICAMP a oportunidade de poder interagir de uma maneira única e direta com o ensino de nível médio, possibilitando a oportunidade de praticar diferentes maneiras de transmissão do conhecimento aos alunos de ensino médio. Ao mesmo tempo possibilita um contato dos alunos de ensino médio com a vida acadêmica e os profissionais que permeiam o ambiente universitário e oferece a esses estudantes a oportunidade de desenvolver senso crítico, compreender a dinâmica da construção e transmissão do conhecimento.

${ }^{5}$ http://www.prp.rei.unicamp.br/pibic/congressos/xxiicongresso/ 


\section{Revista de Arqueologia Pública}

Visa também despertar vocação científica e incentivar talentos potenciais entre estudantes do ensino médio da Rede Pública, mediante sua participação em atividades de pesquisa científica ou tecnológica, orientadas por pesquisador qualificado em seus núcleos de pesquisas (http://www.prp.unicamp.br/pibic-em).

\section{O Programa PIBIC-EM no Núcleo de História e Arqueologia - LAP}

O LAP (Laboratório de Arqueologia Pública) realiza o trabalho com os alunos selecionados no Projeto PIBIC-EM desde 2009. Tem sido uma experiência muito gratificante, tanto para os estudantes envolvidos quanto para o próprio laboratório.

Dentre as várias atividades desenvolvidas merece destaque o programa LAP nas Escolas que aconteceu a partir de aulas expositivas e simulações de escavação arqueológicas com alunos e professores da rede estadual de Campinas, Alfenas e Pelotas. Os alunos PIBIC tiveram contato com o processo de desenvolvimento deste projeto.

Em 2014/2015, foi proposto um novo modelo para o desenvolvimento do programa PIBIC-EM no LAP. Iniciou-se um projeto chamado "Arqueologia, a aventura do conhecimento", sob a coordenação e orientação do Prof. Dr. Pedro Paulo A. Funari, com o objetivo de apresentar aos alunos temas ligados à Arqueologia. Para tanto, os alunos participam ativamente de grupos de discussões com os mais diversos integrantes do laboratório, desde graduandos, passando até por convidados estrangeiros.

O organograma programático foi pensado de tal maneira que buscasse "fortalecer o processo de disseminação das informações e conhecimentos científicos e tecnológicos básicos; desenvolver atitudes, habilidades e valores necessários à educação científica e tecnológica dos estudantes" (http://www.cnpq.br/web/guest/pibic-ensino-medio).

Para isso, o tema escolhido foi "Arqueologia e Alimentação". Por ser a alimentação o fator primordial na rotina diária da humanidade, não apenas uma necessidade básica, mas principalmente, por ser um fator preponderante de cada cultura. $O$ objetivo era fazer um estudo retrospectivo que pudesse oferecer subsídios para uma reflexão sobre o panorama da alimentação em diversas e diferentes culturas. Através da evolução histórica da alimentação mundial, alguns temas como gastronomia, recursos, hábitos e padrões alimentares foram aspectos importantes que auxiliaram a refletir sobre a complexidade e a magnificência que permeiam as relações entre os diversos povos.

Quando se fala em alimentação não há como não pensar na consequência de um tema tão caro aos dias atuais: a fome. Problema de extrema gravidade que atinge milhões 


\section{Revista de Arqueologia Pública}

de pessoas em todo o mundo, em que diversos países subdesenvolvidos enfrentam as mazelas ocasionadas pela subnutrição crônica. As desigualdades econômicas e sociais têm impossibilitado que as populações, principalmente aquelas de países em desenvolvimento, tenham acesso à alimentação. É importante perceber a emergência de decisões políticas que priorizem o desenvolvimento econômico através de uma melhor distribuição de renda e de uma política agrícola, auxiliadas por novas tecnologias.

Primeiramente foram discutidos conceitos básicos e introdutórios acerca do tema da Arqueologia. No presente momento, está sendo trabalhada com os alunos a questão da alimentação nos diversos campos da Arqueologia, perpassando desde a pré-história até o que concerne à Arqueologia Subaquática. Os alunos são incentivados a debater os temas propostos, assim como analisar criticamente os textos e outras fontes que the são indicadas. Ao final de cada encontro, os alunos redigem um pequeno texto sobre as atividades realizadas naquele dia, textos que virão a compor o relatório parcial e final.

Além disso, o laboratório já tem como prática pedir para que os alunos inseridos no PIBIC-EM desenvolvam um projeto final. A exemplo, recordemos o projeto que foi desenvolvido pelos alunos de 2012/2013. Eles produziram uma HQ para crianças, chamado: "De Dinossauros ao Patrimônio: descobrindo a Arqueologia". As crianças foram responsáveis pelo texto, desenho e coloração. O propósito principal desse HQ foi falar acerca do ofício do arqueólogo, questões patrimoniais e sobre o desenvolvimento da Arqueologia no Brasil.

No ano de 2013/2014, os alunos desenvolveram um jogo de tabuleiro chamado: "Trilhas Culturais - Patrimônios Sul-Americanos". Baseado no jogo War, o objetivo principal foi fazer com que os jogadores "descobrissem", de maneira lúdica, quais são os bens materiais tombados pela UNESCO na América do Sul. Nesse projeto, os alunos também foram responsáveis por todas as etapas, recebendo sempre o apoio e eventual ajuda dos participantes do LAP.

\footnotetext{
${ }^{6}$ Disponível online para download no site do LAP (http://www.lapvirtual.org/divulga--o-cient-fica--arqueologia-p-blica.html).

7 Disponível online para download no site do LAP (http://www.lapvirtual.org/divulga--o-cient-fica--arqueologia-p-blica.html).
} 


\section{Revista de Arqueologia Pública}

Espera-se para o final da etapa 2014/2015 a criação de um VLOG ${ }^{8}$ que trate do assunto discutido ao longo desse processo, das discussões focadas na alimentação das diversas culturas e dos diferentes povos. Dessa maneira, outras pessoas poderão não só conhecer o trabalho que foi realizado pelo PIBIC-EM no núcleo de História e Arqueologia da UNICAMP, como também receber dicas e contribuir fazendo comentários, servindo para promoção desse programa que incentiva jovens talentos do ensino médio da Rede Pública.

Esses alunos participaram do XXIII Congresso de Iniciação Científica da Unicamp que aconteceu nos dias 17 a 19 de novembro de 2015 e apresentaram um pôster em homenagem a Paulo Duarte, um dos grandes iniciadores da Arqueologia e da proteção do patrimônio no Brasil. O pôster: "Projeto Acervo Arqueológico do Arquivo Paulo Duarte" foi apresentado pelas alunas Andresa Sales Pupo e Lorvani Karen Diniz que estavam acompanhadas pelo Prof. Dr. Pedro Paulo A. Funari e Dra. Maria Aparecida de A. Almeida.

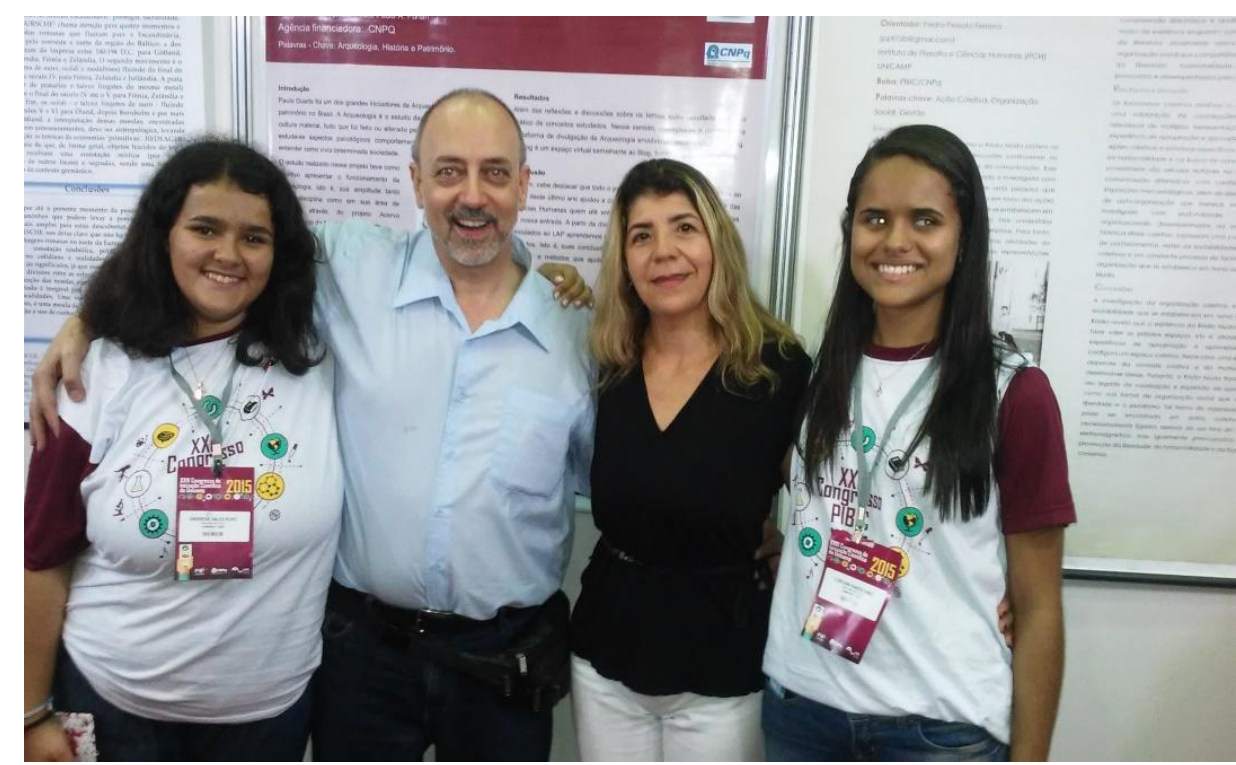

Figura 1: Foto tirada no XXIII Congresso de Iniciação Cientifica da Unicamp 2015. (Foto de Cida Almeida)

\footnotetext{
8 Vlog é a abreviação de videoblog (vídeo + blog), um tipo de blog em que os conteúdos predominantes são os vídeos.
} 
Revista de Arqueologia Pública

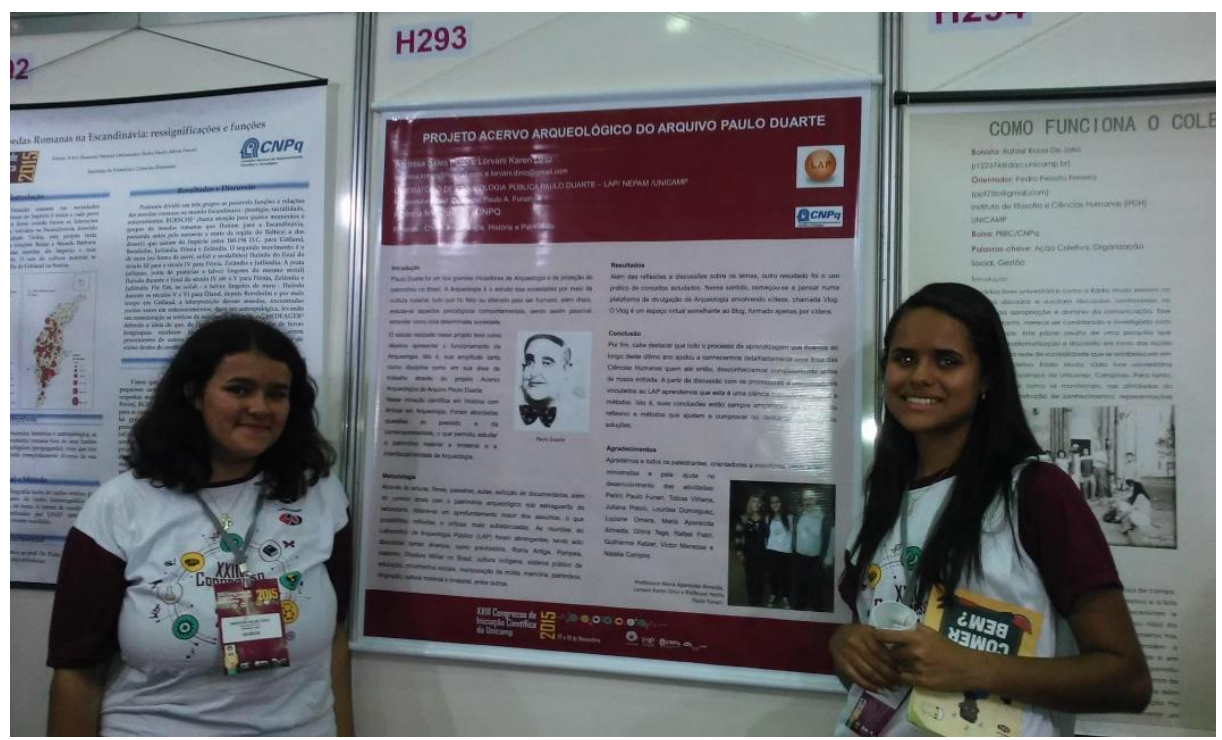

Figura 2: Foto tirada no XXIII Congresso de Iniciação Cientifica da Unicamp 2015. (Foto de Cida Almeida)

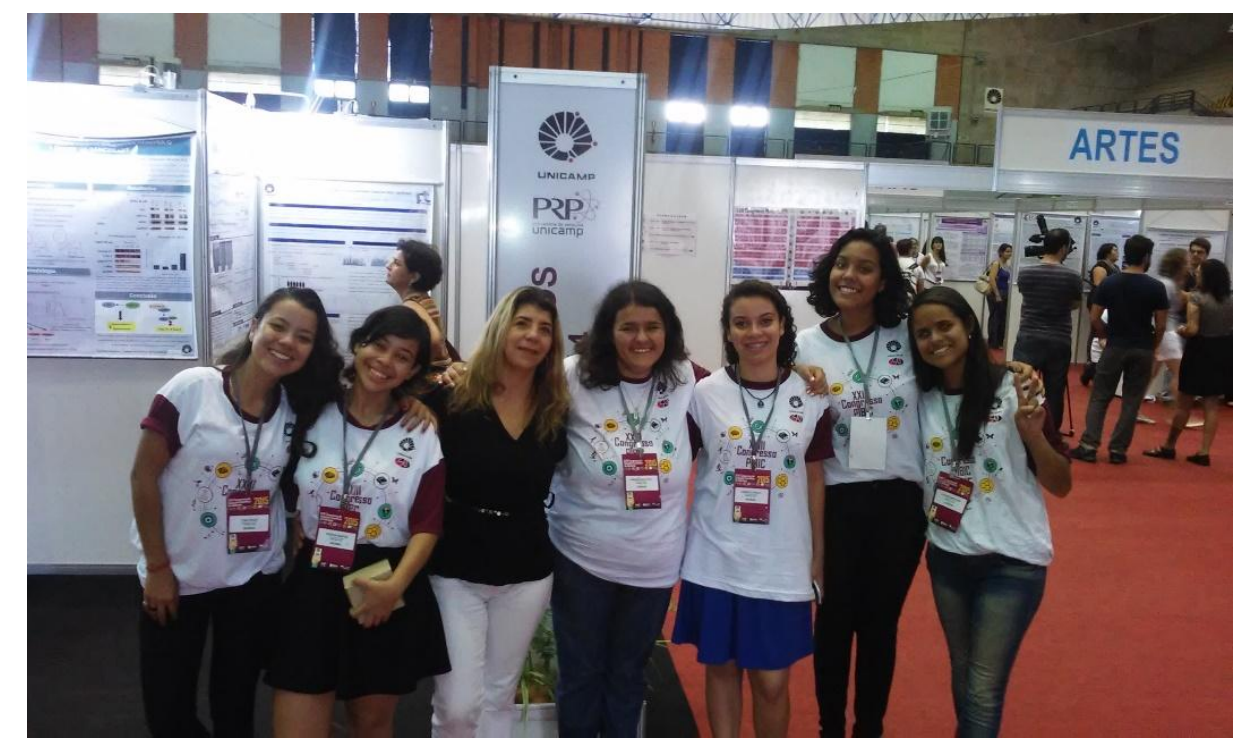

Figura 3: Foto tirada no XXIII Congresso de Iniciação Cientifica da Unicamp 2015. (Foto de Cida Almeida)

Para a etapa que vai de agosto de 2015/2016 o LAP recebeu quatro alunos no programa: Adelaide de Camargo Teixeira, Matheus da Silva Ferreira Barbosa, Stefani Beatris Vieira dos Santos e Beatriz Cristina Carvalho. Conta com a participação de 12 monitores: mestrandos, doutorandos e pós-doutorandos: Luciane Omena, Maria Aparecida de A. Almeida, Tami Ocar, Juliana Poloni, Rafael Patiri, Daniel Grecco, Fernando Pesce, Filipe Silva, Leandro Infantini, Tobias Vilhena de Moraes, Glória Tega, Camila Petini Freitas. 


\section{Revista de Arqueologia Pública}

O projeto está sendo desenvolvido dando sequência ao tema da etapa anterior "Arqueologia e Alimentação". Para isso, foi proposto um cronograma de atividade semanal envolvendo os monitores nas suas respectivas formações e habilidades. O tema está sendo desenvolvido através de aulas expositivas, discussões de documentos escritos e materiais, bibliografia de apoio pertinente ao tema proposto, materiais audiovisuais e reflexões em grupos, de modo que os alunos possam conhecer o que é Arqueologia, o trabalho realizado pela Arqueologia, os hábitos alimentares de diversos povos e a evolução desta através da história e da cultura.

Por fim, vale destacar, que toda aprendizagem que estes alunos adquirem ao longo do processo ajuda no conhecimento detalhado de uma área das Ciências Humanas que, até então, era desconhecida para eles. A partir das discussões com os professores e pesquisadores que colaboraram com o projeto PIBIC-EM vinculado ao LAP, os alunos aprendem que esta é uma ciência baseada em regras e métodos. Isto é, suas conclusões estão sempre amparadas em um estudo reflexivo e métodos que ajudam a comprovar ou descartar determinadas soluções. Tem sido uma experiência muito enriquecedora tanto para os alunos quanto para os colaboradores que participam deste projeto.

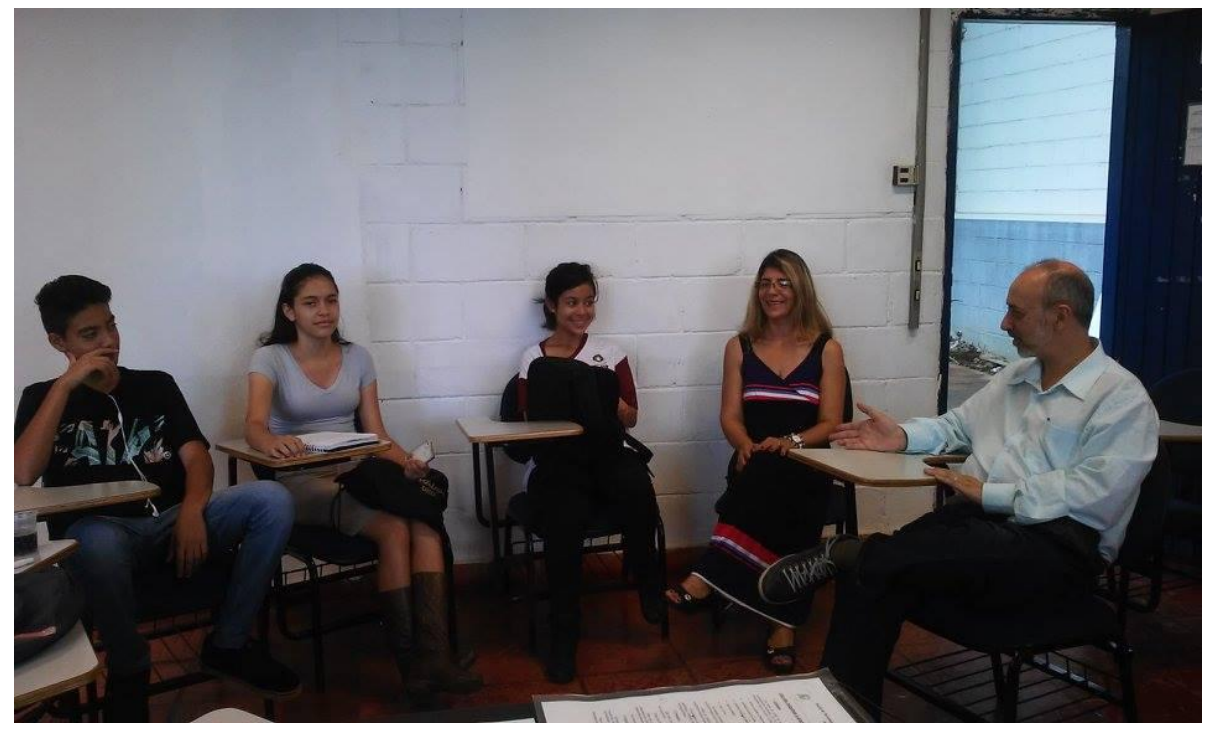

Figura 4: Foto tirada em um encontro do PiBIC-EM em 2015. (Foto de Cida Almeida)

\section{Agradecimentos}

Ao professor Pedro Paulo Abreu Funari e aos que se dispuseram a colaborar com o projeto: Rita Juliana Poloni, Leandro Infantini, Rafael Patiri, Luciane Omena, Tami Coelho Ocar, Daniel Greco Pacheco, Fernando Pesce, Filipe Silva, Tobias, Glória Tega, Camila 


\section{Revista de Arqueologia Pública}

Freitas, Guilherme Ketzer Victor Menezes. Mencionamos também, o apoio institucional da FAPESP, do CNPq, do NEPAM e do LAP UNICAMP.

\section{Referências bibliográficas}

FERNANDES, Marcelo. Arqueologia e educação-programa. Arqueologia e comunidades para crianças e adolescentes no Vale do Jequitinhonha. In: Revista Latino Americana de Ciencias Sociales, Niñez y Juventude, 11.1, Jan. -Jun., pp. 199-216, 2013.

FLANDRIN, Jean-Louis; MONTANARI, Massimo. História da alimentação. São Paulo: Estação Liberdade, 2013.

FREIRE, P. Educação com prática da liberdade. Rio de Janeiro: Paz e Terra, 1980.

FUNARI, P.P.A. Arqueologia. São Paulo, Contexto, 2003.

FUNARI, P. P. A.. A vida cotidiana na Roma Antiga. São Paulo: Annablume, 2003.

FUNARI, P.P.A. ROBRAHN-GONZÁLEZ, E. Editorial, Arqueologia Pública, 1, 2006.

LABORATÓRIO DE ARQUEOLOGIA PÚBLICA PAULO DUARTE. Arqueologia: uma atividade muito divertida. Ilustração Tami Coelho Ocar e Rafael Hakin Patiri. Caluh: Campinas, 2014.

PROUS, André. O Brasil antes dos brasileiros: pré-história do nosso país. Rio de Janeiro: Zahar, 2007.

\section{Sites}

http://www.cnpq.br/web/guest/pibic-ensino-medio

www.prp.unicamp.br/pibic-em

www.lapvirtual.org/divulga--o-cient-fica---arqueologia-p-blica.html 